\title{
Investigation of the homogeneous nucleation of water vapor using a diffusion cloud chamber
}

Richard H. Heist

Fairfield University, rheist@fairfield.edu

Howard Reiss

Follow this and additional works at: https://digitalcommons.fairfield.edu/engineering-facultypubs (C) 1973 The American Institute of Physics.

The final publisher PDF has been archived here with permission from the copyright holder. https://aip.scitation.org/doi/abs/10.1063/1.1680073

\section{Peer Reviewed}

\section{Repository Citation}

Heist, Richard H. and Reiss, Howard, "Investigation of the homogeneous nucleation of water vapor using a diffusion cloud chamber" (1973). Engineering Faculty Publications. 163.

https://digitalcommons.fairfield.edu/engineering-facultypubs/163

\section{Published Citation}

Heist, R. H., \& Reiss, H. (1973). Investigation of the homogeneous nucleation of water vapor using a diffusion cloud chamber. The Journal of Chemical Physics, 59(2), 665-671. doi:10.1063/1.1680073.

This item has been accepted for inclusion in DigitalCommons@Fairfield by an authorized administrator of DigitalCommons@Fairfield. It is brought to you by DigitalCommons@Fairfield with permission from the rightsholder(s) and is protected by copyright and/or related rights. You are free to use this item in any way that is permitted by the copyright and related rights legislation that applies to your use. For other uses, you need to obtain permission from the rights-holder(s) directly, unless additional rights are indicated by a Creative Commons license in the record and/or on the work itself. For more information, please contact digitalcommons@fairfield.edu. 


\title{
Investigation of the homogeneous nucleation of water vapor using a diffusion cloud chamber
}

\author{
Richard H. Heist and Howard Reiss \\ Department of Chemistry, University of California, Los Angeles, Los Angeles, California 90024
}

(Received 9 January 1973)

\begin{abstract}
The temperature variation of the critical supersaturation for water vapor has been measured using a diffusion cloud chamber. By attaching a layer of glass wool to the upper plate of the chamber, the persistent refusal (in previous experiments) of water to wet this plate has been overcome. As a result, for the first time measurements without appreciable scatter have been achieved for water vapor. The resulting temperature dependence agrees reasonably well with that predicted by the classical Becker-Doering-Zeldovitch theory, while the experimental curve is shifted by approximately $8 \%$ from the classical prediction.
\end{abstract}

\section{INTRODUCTION}

One of the more recent developments in the field of vapor-to-condensed phase nucleation has been the application and subsequent refinement of the diffusion cloud chamber. Although this chamber was first proposed by Langsdorf in $1939,{ }^{1}$ it was not until 1956 that Frank and Hertz ${ }^{2}$ first applied it to the study of homogeneous nucleation. They did not continue beyond the initial stages of development, however, and it remained for Katz and Ostermier in $1967,{ }^{3}$ and later Katz in $1970,{ }^{4}$ to refine the method to such an extent that the chamber is now one of the best quantitative methods for studying nucleation processes in a gas. ${ }^{3,4}$

Unlike the piston cloud chamber and supersonic nozzles, the diffusion chamber does not rely on a rapid adiabatic expansion to produce a supersaturated state. Instead, it is a steady state device relying entirely upon quiescent operating conditions. Basically, it consists of two metal plates separated by a glass ring. The lower plate, containing the working fluid, is heated while the upper is cooled. The chamber is designed so that, to a high degree of approximation, one-dimensional, plane parallel diffusion takes place from the surface of the working fluid, through an inert carrier gas, to the upper plate where the vapor molecules condense. By solving the one-dimensional equations for the heat and mass fluxes inside the chamber, one obtains partial pressure and temperature profiles for the chamber interior. ${ }^{4}$ Since the saturation vapor pressure of the working fluid varies exponentially with temperature, and the partial pressure profile is nearly linear, a supersaturated state exists throughout the chamber. By simply varying the temperature difference between the liquid pool and the upper plate, the supersaturation may be varied throughout a large range. By adjusting temperatures so that homogeneous nucleation just occurs (a rate of $\sim 1 \mathrm{~cm}^{3} \cdot \mathrm{sec}^{-1}$ ), the so-called critical supersaturation may be determined.

Although the diffusion cloud chamber compares favorably with both the piston chamber and the supersonic nozzle, ${ }^{3-6}$ it has two important disadvantages. 4 First, since the working fluid must not be allowed to boil, the temperature of the lower plate must remain below the boiling point of the fluid; and since the fluid must not freeze, the upper plate must remain above the fluid's triple point. This limits the working fluid to substances which have boiling points widely separated from their triple points. Second, accurate values for the binary diffusion coefficient and thermal conductivity of the carrier gas-vapor mixture must be known so that temperature and pressure profiles may be determined. These are usually difficult to find.

Water has been, perhaps, the most widely investigated fluid. It is interesting not only from a theoretical point of view but also because of its meteorological significance. Virtually all experimental investigations involving water vapor have been performed using either piston cloud chambers or supersonic nozzles. Reviews of the results of these experiments have been given by Hirth and Pound, ${ }^{5}$ by Stever, ${ }^{7}$ by Wegener and Mack, ${ }^{8}$ and by Wegener. ${ }^{9}$ These reviewers indicate that, while water has been studied extensively, imperfect agreement exists among the results of these inves tigators. Recent measurements by Allen and Kassner ${ }^{10}$ on the homogeneous nucleation of water utilizing a very sophisticated piston cloud chamber indicate that many of the uncertainties which plagued earlier investigators have been removed. ${ }^{5}$ Katz and Ostermier ${ }^{3}$ studied, via the diffusion cloud chamber, the temperature dependence of the critical supersaturation for water vapor, but obtained results which exhibited considerable scatter.

In this paper, we report results for water obtained with a diffusion cloud chamber which, judg- 


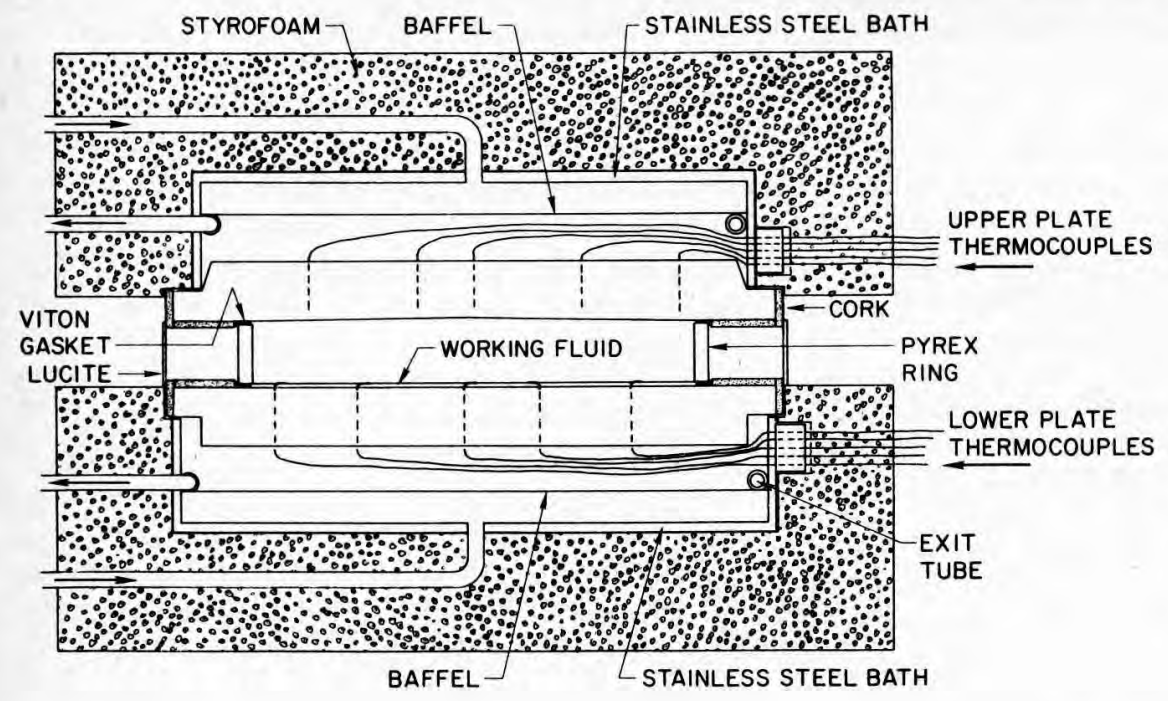

FIG. 1. Diagram of the diffusion cloud chamber. Four nichrome heater wires around the Pyrex ring; two fill ports on the upper plate, and the drain port on the lower plate have been omitted for clarity. The arrows drawn in the circulating system indicate the direction of flow of the heating fluid for the lower plate and the cooling fluid for the upper.

ing from the minimal scatter, appear to provide a reliable picture of the temperature dependence of critical supersaturation.

\section{DESIGN AND OPERATION OF THE CHAMBER}

The basic design of the chamber, with the exception of several modifications, was patterned after Katz's. ${ }^{3,4}$ Since a detailed description of the design and operating features has been given elsewhere, ${ }^{3,4}$ we give a qualitative description of our chamber, noting the modifications.

Figure 1 is a diagram of the device. The upper and lower plates are both $39.4 \mathrm{~cm}$ in diameter, $3.81 \mathrm{~cm}$ thick, and made of solid copper. Copper was chosen because of its high thermal conductivity. The upper plate is machined to have a $1^{\circ} \mathrm{bev}$ el to allow condensate to drain off to the side of the chamber to the glass wall where it returns to the working fluid. If condensate were allowed to fall through the chamber, its quiescent operating state would be destroyed. Both plates have approximately $0.00051 \mathrm{~cm}$ of gold plated on their surfaces, in anticipation of future experiments with corrosive working fluids.

The Py rex ring, which separates the plates, is $3.81 \mathrm{~cm}$ high with an average inside diameter of $28.7 \mathrm{~cm}$ and an average wall thickness of $0.85 \mathrm{~cm}$ (the glass walls are not exactly concentric). The chamber height, measured from the surface of the working fluid to the upper plate at a point one-half the distance from the center to the inside wall of the glass ring, is $4.22 \mathrm{~cm}$, giving a diameter: height ratio of $6.8: 1$. It has been observed ${ }^{3,4}$ that a large diameter-to-height ratio $(\geq 5)$ minimizes wall effects insofar as the diffusion process is concerned. The Pyrex ring is separated from each plate by a Viton gasket. Heater wires wound on the ring are used to keep the inside free of working fluid condensate. The surfaces of each plate are maintained at their respective temperatures by partial immersion in constant temperature baths. In order to minimize circulation paths for the heating and cooling fluids, each bath contains a baffle with approximately 40 small openings (see Fig. 1). These baffles have the effect of creating many small jets of circulating fluid, all of which impinge on the metal plate. The fluid is then forced through an exit tube which runs around the inside circumference of the bath at the surface of each baffle. This arrangement makes it possible to maintain the interior plate surfaces at a very constant and uniform temperature despite the large surface area.

The cooling fluid is methanol and the heating fluid, water. Both baths are made of stainless steel and are thermally insulated with 3 in. of Styrofoam. All exposed copper surfaces are insulated with a layer of cork. A strip of thin Lucite is placed around the cloud chamber to provide a dead air space as insulation for the Pyrex ring. The temperature of the top plate surface is determined by five copper-constantan thermocouples mounted in the plate approximately $0.83 \mathrm{~cm}$ from the inside surface. The temperature of the working fluid surface is measured by five Teflon-coated copperconstantan thermocouples brought into the chamber through the lower plate. The use of Teflon coating was in anticipation of future experiments with corrosive fluids.

The location of the junctions in the Teflon-coated thermocouples was determined via an x-ray photograph. The thermocouples were positioned as nearly as possible so that the junctions were all at the same height. Exact junction positioning was 
difficult, and this contributed to experimental error. All thermocouples were calibrated against a platinum resistance therometer standardized at the National Bureau of Standards. The entire chamber was vacuum tight and lost less than $2 \mathrm{~mm}$ of pressure per day.

In order to observe the nucleation, light from a $650 \mathrm{~W}$ tungsten-halide lamp is heat filtered and focused into the chamber. The light scattered by the small drops as they fall through the light beam is easily visible. In order to avoid errors due to cosmic ray and ion-induced nucleation, ions were swept out by an electric field of approximately 70 $\mathrm{V} / \mathrm{cm}$ maintained across the plates during observation.

Although Katz and Ostermier ${ }^{3}$ used a cloud chamber similar in many respects to the one described above, they were, as indicated earlier, unable to achieve measurements on water vapor, exhibiting a satisfactory degree of reproducibility. This problem arose because water which condensed on the top plate did not wet the metal surface, and hence would not drain off to the sides of the chamber. Large drops formed on the upper plate and subsequently fell through the chamber. These created enough disturbance so that accurate measurements were impossible. Their individual measurements varied by as much as $15 \%-18 \% .^{3}$ We found a rather direct solution to this problem by covering the top plate with a thin (e.g., 0.0102 $\mathrm{cm}$ layer of clean Pyrex glass wool. Since the water wetted the glass wool, a film of condensate formed on the top plate and drained to the sides of the chamber in a satisfactory manner. This simple modification virtually eliminated the problem and enabled us to achieve quantitative measurements of critical supersaturations as a function of temperature.

The partial pressure and temperature profiles inside the chamber were calculated in the manner of Katz. ${ }^{3,4}$ The equations for the mass and heat fluxes in the chamber are $\mathrm{a}^{3,4}$

$$
\begin{array}{r}
d P / d z=\left[\alpha P\left(P-P_{t}\right) / T P_{t}\right] \\
\times(d T / d z)+\left[\left(P-P_{t}\right) L / T^{s} D_{a b}^{0}\right], \\
d T / d z=\lambda^{-1}\left(-q+L\left\{H_{a}+\left.\left(H_{c}-H_{a}\right)\right|_{T_{1}} \mid T_{1}\right.\right. \\
\left.\left.+\left[\alpha R T\left(P_{t}-P\right) / P_{t}\right]\right\}\right),
\end{array}
$$

where $P$ is the partial pressure of the vapor, $P_{t}$ the total pressure, and $T$ the absolute temperature. $\alpha$ is a thermal diffusion ratio defined to be $k_{T} / X_{a} X_{b}$, where $k_{T}$ is the thermal diffusion factor and $X_{a}$ the mole fraction of species $a ; z$ is a reduced chamber height given by $Z / h, h$ being the actual chamber height, and $0 \leq z \leq 1 . \quad D_{a b}^{0}$ is a constant related to the binary diffusion coefficient, $D_{a b}$, in the following way:

$$
D_{a b}=D_{a b}^{0} R T^{s+1} / P_{t},
$$

where $T$ is the temperature and $s \approx 0.75$. $L$ is a reduced molar flux for vapor component $a$ given by $L_{a} h, \lambda$ is the thermal conductivity of the gas mixture, $H_{a}$ is the enthalpy of the vapor, $H_{c}$ is the heat of condensation, $T_{1}$ is the upper plate temperature, $q$ a reduced heat flux given by $Q h$, and $R$ is the gas constant. The method for solution of these equations is given elsewhere. ${ }^{3,4}$ An example of the partial pressure and temperature profiles along with the variation of supersaturation throughout the chamber is given in Fig. 2.

\section{EXPERIMENTAL PROCEDURE AND RESULTS}

Although the diffusion cloud chamber is selfcleaning with respect to particulate matter such as dust, it is important that impurities, particularly volatile ones, be kept to a minimum. Both the metal plates and Pyrex ring were cleaned with soap and water, acetone and cleaning acid, and then rinsed thoroughly with triply distilled water. The Viton gaskets were washed with soap and water, cleaned with ethanol, and then boiled in triply distilled water to leach out any water soluble impurities. The thin layer of Pyrex wool was soaked in cleaning acid and then thoroughly rinsed and dried. It was then applied to the upper plate and lightly sprayed with triply distilled water. The surface forces of the water were sufficient to hold the wool in place. The thermocouples on the lower plate were positioned so that the center of each (Teflon insulation) was $2 \mathrm{~mm}$ from the plate surface. This was accomplished by using a $2 \mathrm{~mm}$ thick Teflon spacer and adjusting each thermocouple so that its center coincided with the top of the spacer. The chamber was assembled and flushed repeatedly with either hydrogen or helium, the two carrier gases used for this investigation. Triply distilled, degassed water was then introduced in such a way as to avoid contamination from the external atmosphere. The chamber was filled and drained and then filled once more to flush any impurities that might have entered prior to assembly. Care was exercised during draining that no contamination from the outside environment occurred. After the water level had been adjusted as nearly as possible to $2 \mathrm{~mm}$, the experiment began.

A typical experimental sequence for one run proceeded as follows. The lower plate was heated and the upper cooled until nucleation was observed. The wall heating was adjusted so that any condensate on the inside of the glass just disappeared. This generally required $55-60 \mathrm{~W}$. The electric field was turned on and final adjustments 


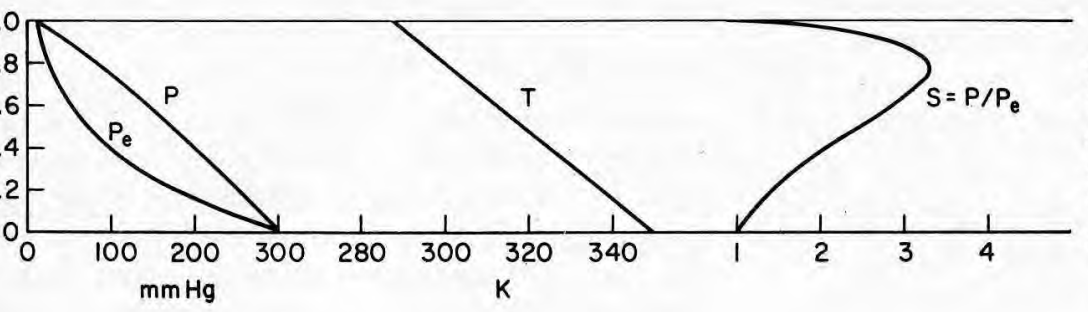

FIG. 2. Variation of the partial pressure, $P$, saturation vapor pressure, $\boldsymbol{P}_{e}$, temperature, $T$, and supersaturation, $S$, as a function of reduced chamber height. These plots represent actual data from Expt. 17.

of the temperature gradient were made until a rate of approximately $1-3 \mathrm{drops} / \mathrm{cm}^{3} / \mathrm{sec}$ was established. Heating and cooling systems were sensitive enough so that variations in temperature difference of a few hundredths of a degree could be made with ease. When the proper rate was established, the system was left alone for approximately $45 \mathrm{~min}$ to insure complete equilibration. After this the rate was again checked. If it had not changed, the chamber pressure was measured, the temperatures of both plates determined, and then the pressure was remeasured to insure against any significant changes. After these measurements, the temperature of each plate was changed by a few degrees, the pressure readjusted, and the sequence repeated. This was continued until the entire accessible temperature range had been investigated. Thermocouple measurements were made using a Leeds and Northrop K-4 potentiometer coupled to a Keithley Model $155 \mu \mathrm{V}$ null detector. The output of the null detector was then displayed on an Esterline Angus chart recorder.

During the course of these experiments, it was determined that the temperature variation over the upper plate surface averaged approximately $0.05{ }^{\circ} \mathrm{C}$, while the variation of the water surface temperature over the lower plate surface was generally $0.1-0.3^{\circ} \mathrm{C}$. It was observed that the lower the temperature the more constant the overall plate temperature. The temperature readings from each lower plate thermocouple were found to oscillate about an average value by as much as several tenths of a degree. Every thermocouple reading was extended over a long enough period to determine this average value. The upper plate thermocouples did not oscillate, and remained essentially constant during each reading.

Since the film of condensate was of finite thickness, a correction had to be introduced for the temperature drop from the film to the plate surface. $\mathrm{Katz}^{4}$ has given an approximate relation for estimating the film thickness on the upper plate. We used this relation estimating the thickness of a film of water assuming it had wet the plate. To this, we added the glass wool dimension to obtain an approximate film thickness. The correction generally varied between 0.1 and $0.3{ }^{\circ} \mathrm{C}$.

In Table I, the three parameters obtained from experiment, surface temperature of the working fluid, $T_{0}$, upper plate film temperature, $T_{1}$, and total pressure, $P_{t}$, are listed for each of the experiments. In Table II, all of the additional information necessary to compute partial pressure and temperature profiles, temperature drop across the condensate film on the upper plate, and the variation of the critical supersaturation with temperature as predicted by the conventional theory ${ }^{4}$ are given. Figure 3 exhibits plots of the individual experiments. The experimental variation of the critical supersaturation with temperature is the envelope of all of these curves. ${ }^{4}$

TABLE I. Experimental data for the various chamber states: $T_{0}$ is the surface temperature of the working fluid, $T_{1}$ is the film temperature on the upper plate, and $P_{t}$ is the total pressure inside the cloud chamber.

\begin{tabular}{rcrr}
\hline $\begin{array}{c}\text { Experiment } \\
\text { No. }\end{array}$ & $T_{0}\left({ }^{\circ} \mathrm{K}\right)$ & $T_{1}\left({ }^{\circ} \mathrm{K}\right)$ & $P_{t}(\mathrm{~mm})$ \\
\hline \multicolumn{4}{c}{ Water-helium } \\
1 & 341.59 & 280.07 & 737.7 \\
2 & 346.91 & 285.23 & 768.6 \\
3 & 351.73 & 290.63 & 728.4 \\
4 & 354.67 & 294.64 & 729.6 \\
5 & 358.05 & 298.02 & 757.7 \\
6 & 364.57 & 305.33 & 753.6 \\
7 & 337.37 & 276.53 & 736.5 \\
8 & 335.25 & 273.86 & 775.0 \\
9 & 340.30 & 278.31 & 798.8 \\
10 & 336.04 & 274.14 & 781.5 \\
11 & 356.63 & 293.32 & 1102.7 \\
12 & 356.77 & 294.94 & 1044.8 \\
13 & 362.91 & 301.39 & 1101.2 \\
14 & 365.87 & 304.83 & 1038.8 \\
& Water-hydrogen & \\
15 & 339.56 & 278.19 & 779.2 \\
16 & 344.54 & 282.31 & 818.5 \\
17 & 349.24 & 287.94 & 774.0 \\
18 & 352.19 & 290.17 & 888.7 \\
19 & 355.36 & 292.52 & 1070.7 \\
20 & 335.16 & 273.63 & 773.6 \\
\hline \hline
\end{tabular}


TABLE II. Expressions for the thermal conductivity, $\lambda$, saturation vapor pressure, $P_{e}$, surface tension, $\sigma$, viscosity, $\eta$, and density $d$; and values for the molecular weight, $M$, molar heat capacity at constant pressure, $C_{p}$, thermal diffusion ratio, $\alpha$, binary diffusion coefficient, $D_{a b}$, and the binary diffusion coefficient temperature dependence, $s{ }^{a}$

Water:

$$
\begin{aligned}
\lambda= & -1.6487 \times 10^{-5}+1.9895 \times 10^{-7} T^{\mathrm{b}} \\
\log _{10} P_{e}= & 19.301142-2892.3693 / T-2.892736 \log _{10} T \\
& -4.9369728 \times 10^{-3} T+5.606905 \times 10^{-6} T^{2} \\
& -4.645869 \times 10^{-9} T^{3}+3.7874 \times 10^{-12} T^{4 \mathrm{c}} \\
\sigma= & 93.6635+0.009133 T-0.000275 T^{2 \mathrm{~d}} \\
\eta_{1 \mathrm{iq}}^{-1}= & 2.1482\left\{(T-281.585)+\sqrt{8078.4+(T-281.585)^{2}}\right\}-120^{\mathrm{e}} \\
\eta_{\text {gas }}= & 183.5 \times 10^{-7}\left[T^{1.5} /(T+668.3)\right]^{\mathrm{f}} \\
d= & 1.0-\left[(T-277.1363)^{2}(T+15.7914) / 508929.2(T-205.02037)\right]^{\mathrm{g}} . \\
M= & 18.015 ; \quad C_{p}=8.0^{\mathrm{h}} ; \alpha=0.3^{\mathrm{i}} ; \mathrm{H}_{2} D_{a b}=0.7516^{\mathrm{j}} ; \\
s= & 0.712^{\mathrm{j}} ; \quad{ }_{\mathrm{He}} D_{a b}=0.719^{\mathrm{k}} ; s=0.75^{\mathrm{k}}
\end{aligned}
$$

Helium:

$$
\begin{aligned}
\lambda & =7.376974 \times 10^{-5}+1.139222 \times 10^{-6} T-6.343536 \times 10^{-10} T^{2 \mathrm{~b}} \\
\eta & =145.5 \times 10^{-7}\left[T^{1.5} /(T+74.1)\right]^{1} \\
M & =4.0026
\end{aligned}
$$

Hydrogen:

$$
\begin{aligned}
\lambda & =-5.468234 \times 10^{-5}+2.137513 \times 10^{-6} T-1.697643 \times 10^{-9} T^{2 \mathrm{~b}} \\
\eta & =64.9 \times 10^{-7}\left[T^{1.5} /(T+74)\right]^{\mathrm{m}} \\
M & =2.01594
\end{aligned}
$$

${ }^{2} \lambda$ in $\mathrm{cal} /(\mathrm{cm} \cdot \mathrm{sec} \cdot \mathrm{K}) ; P_{e}$ in $\mathrm{mm} ; \sigma$ in erg $/ \mathrm{cm}^{2} ; \eta$ in poise; $C_{p}$ in cal $/(\mathrm{mole} \cdot \mathrm{K}) ; D_{a b}$ in $\mathrm{cm}^{2} / \mathrm{sec}$ at $273.16{ }^{\circ} \mathrm{K} ; \alpha \cdot$ and $s$ are dimensionless; and $T$ in ${ }^{\circ} \mathrm{K}$.

'Thermophysical Properties Research Center, Data Book (Purdue University, Lafayette, Ind., 1970 ), Vol. 3.

'Landolt-Börnstein Zahlenwerte und Funktionen aus Physik-Chemie-Astronomie-Geophysik-Technik (Springer-Ver lag, Berlin, 1960), Vol. 2, Pt. 2a, p. 32.

${ }^{\mathrm{d}}$ Footnote (c), Vol. 2, Pt. 3, p. 421 (1956).

e"Properties of Ordinary Water-Substance," compiled by E. Ernest Dorsey, National Bureau of Standards, Washington, D. C. (1958).

${ }^{\mathbf{I}}$ The expression for the viscosity of water vapor was obtained from the data of $\mathrm{H}$. Braune and R. Linke, Z. Physik. Chem. A148, 195 (1930); and from the data in the International Critical Tables, (1929), Vol. 5, p. 4 for the temperature range of $275-680^{\circ} \mathrm{K}$.

${ }^{\text {g}} \mathrm{L}$. W. Tilton and J. K. Taylor, J. Res. Nat. Bur. Std. 18, 205 (1937).

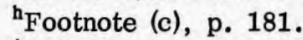

${ }^{1}$ The thermal diffusion ratio is not known for the water-helium and water-hydrogen mixtures, so it is approximated as $\alpha=0.3 .^{3,4}$ The resulting calculated supersaturation curves are quite insensitive to $\alpha$ since variations of $\pm 33 \%$ produce changes less than $1 \%$ in the calculated maximum supersaturation.

${ }^{j}$ E. T. Nelson, J. Appl. Chem. 6, 286 (1956).

${ }^{\mathrm{k}_{E}}$. N. Fuller, P. D. Schettler, and J. C. Giddings, Ind. Eng. Chem. 58, 19 (1966).

${ }^{1}$ The expression for the viscosity of helium gas was obtained from the data of Onnes and Weber, Vers. Kon. Akad. van Wetenschappen, Amsterdam 21, 1385 (1913) for the temperature range of $200-450^{\circ} \mathrm{K}$.

m The expression for the viscosity of hydrogen gas was obtained using data from the International Critical Tables (McGraw-Hill, New York, 1929), Vol. 5, p. 5 for the temperature range $270-400^{\circ} \mathrm{K}$.

The solid curves are data using helium as the carrier gas, while the dashed curves refer to hydrogen as carrier gas. The solid line is the variation of the critical supersaturation predicted by the conventional theory. This prediction is obtained by setting the rate, $J$, equal to $1 \mathrm{~cm}^{-3} \cdot \mathrm{sec}^{-1}$ in the following relation and solving iteratively for supersaturation, $S$, as a function of temperature,

$$
J=\frac{a}{d}\left(\frac{2 N_{0}^{3} \sigma M}{\pi}\right)^{1 / 2}\left(\frac{S P_{e}}{R T}\right)^{2} \exp \left[-\frac{16 \pi N_{0}}{3(\operatorname{lnS})^{2}}\left(\frac{M}{d}\right)^{2}\left(\frac{\sigma}{R T}\right)^{3}\right] \text {. }
$$




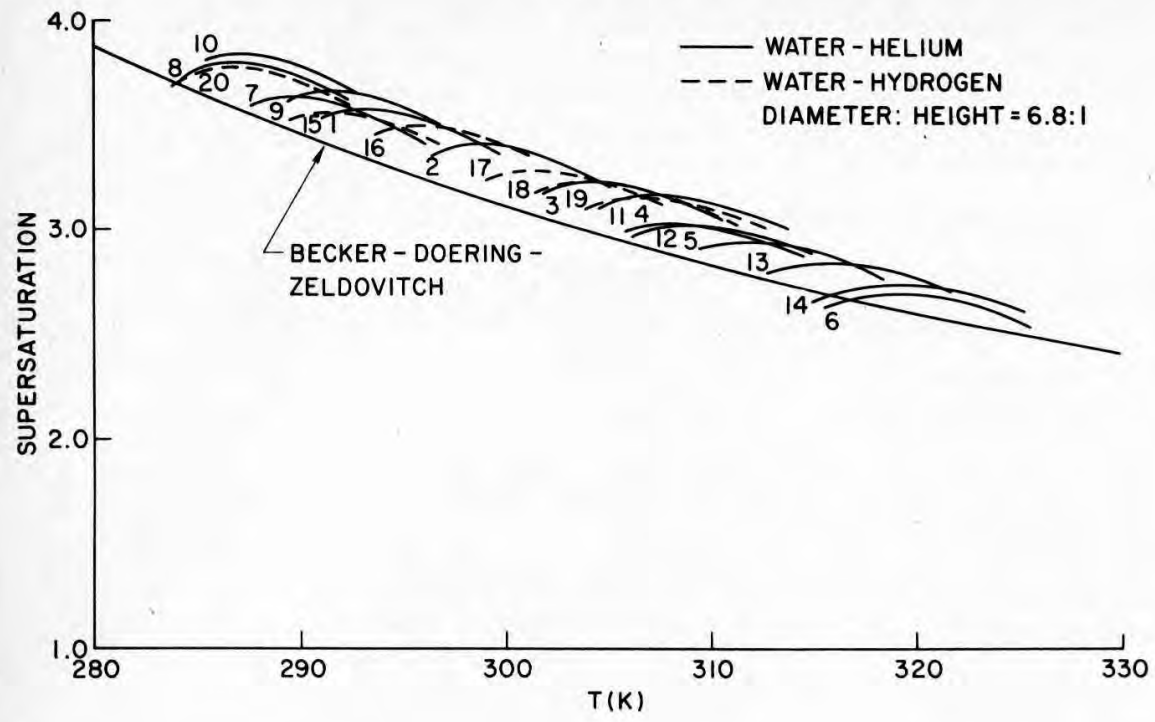

FIG. 3. The variation of the critical supersaturation of water vapor as a function of temperature. The solid curve is the variation predicted by the BeckerDoering-Zeldovitch theory, and the envelope of the numbered curves represents the measured variation. The dashed curves denote experiments using hydrogen as a carrier gas, while the solid curves denote experiments using helium as the carrier gas.
Here $a$ is the condensation coefficient, $d$ the density, $N_{0}$ Avogadro's number, $\sigma$ the surface tension, $M$ the molecular weight, $S$ the supersaturation, $P_{e}$ the saturation vapor pressure, $R$ the gas constant, and $T$ the absolute temperature. The value of the condensation coefficient was taken as unity. The actual experimental envelope has been omitted for the sake of clarity.

\section{DISCUSSION}

As is illustrated in Fig. 3, the scatter in the earlier work of Katz and Ostermier ${ }^{3}$ has been virtually eliminated. This is an interesting point , since such large deviations ${ }^{3}$ have been interpreted by some authors ${ }^{10}$ as indicative of an irregular buildup of ions in the chamber due to large deviations in the normal cosmic ray activity. It should also be noted that the experimental supersaturation curve is virtually parallel to, and approximately $8 \%$ higher, than that predicted by conventional theory. Figure 3 also illustrates that there is no measurable dependence on the carrier gas. This is true as long as the gas is light enough to maintain the proper density gradient. ${ }^{3,4}$ The power supplied to the glass wall to remove the water condensation was varied from 50 to $80 \mathrm{~W}$ with virtually no change in the resulting experimental curves. In order to determine whether or not the applied electric field was sufficient to render the cloud chamber ion free, a rate of about $1-3 \mathrm{drops} / \mathrm{cm}^{3} \cdot \mathrm{sec}^{-1}$ was established with a field of $70 \mathrm{~V} / \mathrm{cm}$, and then examined after the applied field had been doubled. The two rates were virtually indistinguishable.

Unless sufficient carrier gas is present in the chamber to maintain the proper density gradient to prevent convection, the quiescent state of the chamber is disrupted. $\mathrm{Katz}^{3,4}$ observed that if $P_{t} / P_{e}$ is significantly less than 2.5 , where $P_{t}$ is the total chamber pressure, and $P_{e}$ is the saturation vapor pressure of the working fluid at the temperature of the working fluid surface, the resulting supersaturation curves become increasingly unreliable. Experiments 5, 6, 13, and 14 were recorded with $P_{t} / P_{e}<2.5$, the smallest value being 1.3 for Expt. 6 ; the reason being that at these higher temperatures, $P_{t}$ could not be increased more than 1.4 times atmospheric pressure for fear of rupturing the Pyrex ring. The effect of having a pressure ratio of $<2.5$ in the chamber is examined in Expts. 3, 4, 12, and 18. Experiment 18 was run with $P_{t} / P_{e}=2.6$ using hydrogen as a carrier gas, while Expt. 3 was run under nearly identical conditions except $P_{t} / P_{e}=2.2$ and helium was used as a carrier gas. The resulting supersaturation curves are virtually identical. In addition, Expt. 4 was run with $P_{t} / P_{e}=1.9$, and Expt. 12 under similar conditions with $P_{t} / P_{e}=2.5$. The resulting supersaturation curves are in good agreement. These results seem to indicate that even if $P_{t} / P_{e} \cong 1.9$, the supersaturation curves are still fairly reliable.

$\mathrm{Katz}^{4}$ has given a detailed analysis of both the validity of the assumptions necessary to derive Eqs. (1) and (2), and of the inaccuracies introduced due to the experimental uncertainties inherent in the values of the parameters required. The thermal conductivity of the water vapor-carrier gas mixture is felt to be the most unreliable. The thermal conductivity of the mixture was not avail- 
able and was approximated using the Wassiljewa equation, ${ }^{11}$

$$
\lambda(T)=\frac{\lambda_{a}(T)}{1+A_{a b}\left(X_{b} / X_{a}\right)}+\frac{\lambda_{b}(T)}{1+A_{b a}\left(X_{a} / X_{b}\right)},
$$

where $\lambda_{a}$ and $\lambda_{b}$ are the thermal conductivities of the pure water vapor and pure carrier gas, respectively, given in Table II, and $X_{a}$ and $X_{b}$ are the corresponding mole fractions. Expressions for $A_{a b}$ and $A_{b c}$ for binary mixtures have been given by Lindsay and Bromley ${ }^{12}$ and by Mason and Saxena. ${ }^{13}$ The method of Lindsay and Bromley is used since they have calculated thermal conductivities of water-air mixtures which agree fairly well with experiment. ${ }^{12}$ Both approaches give essentially identical answers for binary mixtures of nonpolar molecules, ${ }^{4,11-13}$ but the Mason-Saxena method is expected to be less reliable for polar molecules. ${ }^{11,13}$

The value of the binary diffusion coefficient, $D_{a b}$, and its temperature dependence, $s$, for the waterhydrogen mixture is obtained from Nelson's earlier work. ${ }^{14}$ Experimental values for the water-helium mixture could not be found, so the binary diffusion coefficient is calculated following the procedure of Fuller et al. ${ }^{15}$ This approach is felt to be reasonable since the resulting supersaturation curves, using both the experimental and calculated values of $D_{a b}$ for the water-hydrogen system, differed by less than $1 \%$. The supersaturation curves were relatively insensitive to small changes (several percent) in the values chosen for $D_{a b}, s$, and $\lambda$.

Although we do not attempt to validate the conventional nucleation theory, it must be noted, as shown in Fig. 3, that the Becker-Doering-Zeldovitch theory does provide a fair $(8 \%)$ approximation to the experimental results. It should also be remarked again that probably, for the first time, reliable and reproducible homogeneous nucleation data for water vapor has been obtained in a quiescent steady state measurement. Since water is of primary importance in atmospheric problems, we consider this to be an important result.

\section{ACKNOWLEDGMENTS}

This research is being supported by the National Science Foundation under Grant No. GP-28722X.

The authors wish to express appreciation to Professor J. L. Katz of the Clarkson College of Technology for a number of illuminating discussions regarding the design of the cloud chamber, and for supplying a copy of the computer program necessary to compute the pressure and temperature profiles within the chamber. The authors also wish to acknowledge the assistance of Mr. Steven Thayer during the initial phase of the experimental program. One of the authors (RHH) is especially grateful to Mr. Erik Olsen for his expert assistance through all phases of the cloud chamber construction.

${ }^{1}$ A. Langsdorf, Rev. Sci. Instrum. 10, 91 (1939).

${ }^{2}$ J. P. Frank and H. G. Hertz, Z. Phys. 143, 559 (1956).

${ }^{3}$ J. L. Katz and B. J. Ostermier, J. Chem. Phys. 47, 478 (110).

${ }^{4}$ J. L. Katz, J. Chem. Phys. 52, 4733 (1970).

${ }^{5}$ J. P. Hirth and G. M. Pound, Condensation and Evaporation (Pergamon, New York, 1963), Chap. 2.

${ }^{6}$ R. P. Andres, Nucleation, edited by A. C. Zettlemoyer (Dekker, New York, 1969), Chap. 2.

${ }^{7}$ H. G. Stever, Fundamentals of Gas Dynamics (Princeton U. P., Princeton, NJ, 1958), Vol. 3

${ }^{8}$ P. P. Wegener and L. M. Mack, Adv. Appl. Mech. 5, 412 (1958).

${ }^{9}$ P. P. Wegener, Prog. Astronaut. Aeronaut. 15, 701 (1964).

${ }^{10}$ L. B. Allen and J. L. Kassner, Jr., J. Colloid Interface Sci. 30, 81 (1969).

${ }^{11}$ R. C. Reid and T. K. Sherwood, The Properties of Gases and Liquids (McGraw-Hill, New York, 1966), 2nd ed., Chap. 10.

${ }^{12}$ A. L. Lindsay and L. A. Bromley, Ind. Eng. Chem. 42, 1508 (1950).

${ }^{13}$ E. A. Mason and S. C. Saxena, Phys. Fluids 1, 361 (1958).

${ }^{14}$ E. T. Nelson, J. Appl. Chem. 6, 286 (1956).

${ }^{15}$ E. N. Fuller, P. D. Schettler, and J. C. Giddings, Ind. Eng. Chem. 58, 19 (1966). 